\title{
Comportamiento sísmico de presas de relaves construidas aguas abajo y línea central
}

\author{
Seismic behaviour of tailing dams built by the downstream and centreline methods
}

Fecha de entrega: 15 de diciembre 2016

Fecha de aceptación: 25 de abril 2017

\section{Abraham Figueroa, David Solans, Christian Gonzalez y José Campaña}

Arcadis Chile, Antonio Varas 621, Providencia, Chile, abraham.figueroa@arcadis.cl, david.solans@arcadis.cl, christian.gonzalez@arcadis.cl,jose.campana@arcadis.cl

El crecimiento de la tasa de tratamiento de mineral durante las últimas décadas ha requerido un incremento en la capacidad de los depósitos de relaves. Consecuentemente, la altura y el volumen de las presas de arenas diseñadas han alcanzado dimensiones sin precedentes, debido a limitantes ambientales de acuerdo a la normativa vigente. En este contexto el diseño de presas de arena según línea central es una alternativa para reducir el volumen de arena requerido sin afectar la estabilidad global de la presa. Este método de crecimiento ha sido aplicado satisfactoriamente con presas de gran altura en paises sísmicos, tales como Canadá y recientemente en Perú. El presente artículo presenta la modelación numérica en dos dimensiones de presas de relaves de gran altura sometidas a un sismo severo. Para este análisis se ha considerado una sección característica del crecimiento aguas abajo en un caso, y en un segundo caso esta sección modifica su crecimiento a línea central a partir de cierta cota. Se compara la respuesta sísmica de ambas secciones en cuanto a deformaciones post sísmicas, aceleraciones del coronamiento y amplificación respecto a campo libre, pérdida de revancha operacional, entre otros.

Palabras clave: presas de relaves, comportamiento sísmico, modelación numérica
The growth of ore treatment rates during the last decades has required an increase in the tailings impoundment capacity. Consequently, the height and volume of the designed sand dams have reached unprecedented dimensions. This has been caused by environmental constraints according to current regulations. In this context, the sand dam design with the centreline method is an alternative to reduce the sand volume without affecting the global dam stability. This method of construction has been applied successfully in seismic countries with high tailings dams such as Canada and more recently in Peru. This article presents 2D numerical modelling of high tailings dams under a strong seismic event. For this analysis it has been considered a standard section of dam built by downstream in one case. A second case considers the same section modifying its growth method to centreline from a certain height. The seismic response for both methods and the effect of variation in the upstream slope, in terms of seismic deformations, crest accelerations, amplification respect to free field and among others, are compared.

Keywords: tailings dams, seismic behaviour, numerical modelling

\section{Introducción}

Las presas de arenas de relave han sido ampliamente adoptadas en la industria minería metálica, y específicamente en las minas de cobre donde el continuo crecimiento en las tasas de producción durante los últimos 20 años ha requerido de grandes depósitos de relaves a bajos costos. La construcción de presas de relaves puede desarrollarse mediante tres métodos, referidos normalmente como: aguas arriba, aguas abajo y línea central. En Chile, un país altamente sísmico la construcción de presas de relaves mediante el método aguas arriba no está permitido, debido a la vulnerabilidad de las presas ante la eventual licuefacción de las arenas. Posterior al sismo de 1965, donde se produce la falla de la presa El Cobre $N^{\circ} 1$ 
con más de 200 víctimas, las presas de relaves en Chile han sido construidas mayormente mediante el método de aguas abajo. Las presas de gran altura construidas con este método, durante las 5 décadas siguientes, han mostrado una adecuada respuesta frente a sismos de gran intensidad.

Una escasa incursión se ha efectuado en Chile respecto al crecimiento del tipo línea central. Un caso documentado corresponde a la Presa de relaves Torito con $78 \mathrm{~m}$ de altura, la cual cambió de un crecimiento aguas abajo a uno línea central (Ortiz et al., 2012), presentando hasta la fecha un adecuado comportamiento. Como referencia de presas de línea central de gran altura se pueden mencionar la presa Cerro Verde en Perú, que alcanzaría una altura máxima de $260 \mathrm{~m}$ (Obermayer y Alexieva, 2011), además de presas ubicadas en Norteamérica, Thompson Creek en Canadá (golder.com) que alcanzaría una altura de $220 \mathrm{~m}$ y la de Highland Copper Valley en EEUU (Scott et al., 2007) que alcanzaría una altura máxima de $169 \mathrm{~m}$.

En este contexto, el presente artículo pretende evaluar y comparar, mediante modelación numérica, el comportamiento sísmico de una presa construida con los métodos aguas abajo y línea central.

\section{Presas de arenas de relave del tipo aguas abajo y línea central}

Las presas de arenas de relaves se construyen con una parte del relave minero. En general, el relave es sometido a un proceso mecánico de clasificación a través de un ciclón, donde el relave es fraccionado en una porción gruesa, denominada genéricamente como arenas y, otra más fina, generalmente denominada como lama. En este sentido, la disponibilidad de arenas para la conformación de la presa está condicionada por las características del relave y las exigencias establecidas para la arena. Muchas veces se requieren cantidades importantes de arena que no se encuentran siempre disponibles.

Según se mencionó anteriormente, existen tres tipos básicos de sección transversal de presas de relaves construidas mediante depositación hidráulica denominados, de acuerdo a su método de crecimiento, como aguas arriba, aguas abajo y línea central, tal como se ilustra en la Figura 1.

En regiones sísmicas el método de crecimiento aguas abajo ha sido la alternativa más empleada, frente al insatisfactorio comportamiento que han tenido las presas aguas arriba. La mayor ventaja de este tipo de crecimiento es que la presa se construye cuando ya se ha preparado el suelo de fundación, siendo posible instalar drenes en la base del muro, que facilitan el drenaje, así como la compactación de las arenas que aumentan la rigidez del cuerpo resistente. Sin embargo, las presas aguas abajo requieren una mayor cantidad de arena para su conformación, situación que a veces no resulta compatible con las tasas de crecimiento. En esta situación la construcción de presas con crecimiento de línea central se ha adoptado como una alternativa factible (Obermayer y Alexieva, 2011).

\section{Descripción del caso de estudio}

Tal como el caso de la presa de relaves el Torito (Ortiz et al., 2012), el caso de estudio corresponde a una sección que considera hasta cierta altura un crecimiento aguas abajo, para luego desarrollar un crecimiento en línea central en un caso, o mantener su crecimiento aguas abajo en un segundo caso. Para ambos casos se tiene una altura máxima de $180 \mathrm{~m}$, coronamiento de $15 \mathrm{~m}$, un talud aguas abajo de 1:3.5 V:H y un talud aguas arriba de 1:2 V:H, según se ilustra en la Figura 2.

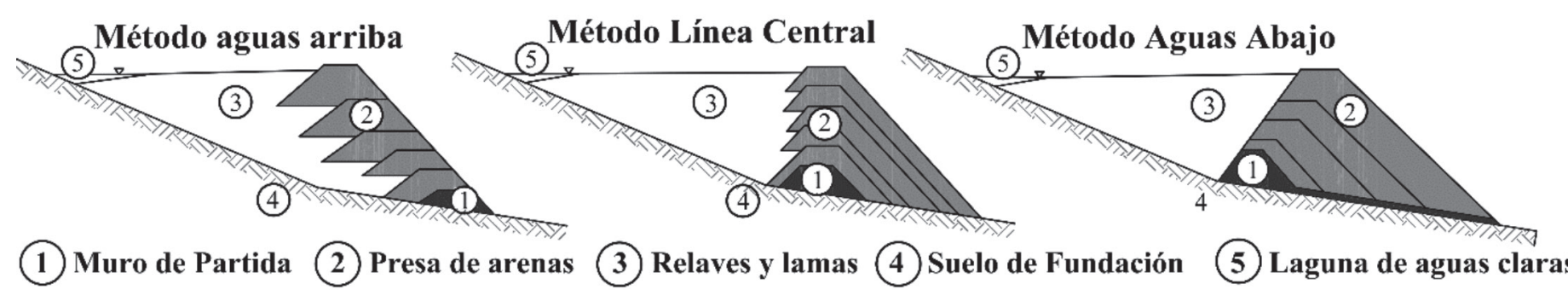

Figura 1: Secciones típicas de presas de relaves según su método de crecimiento 

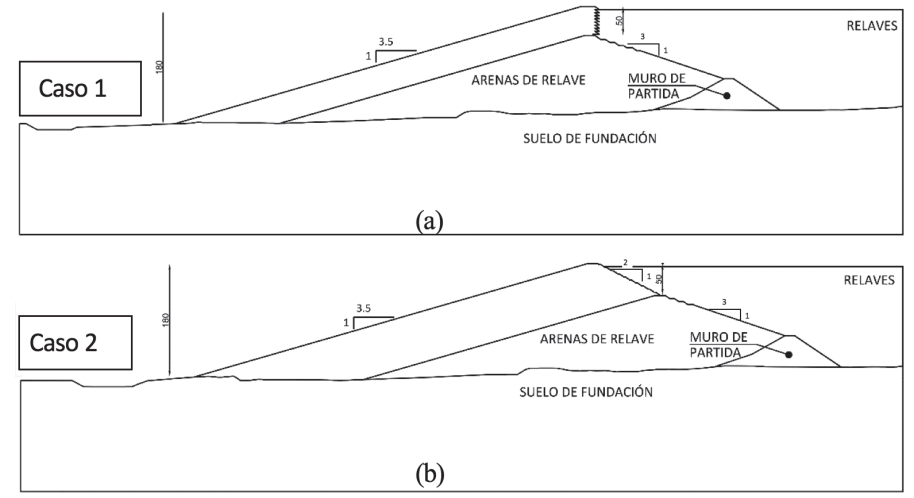

Figura 2: Secciones de análisis: a) Caso 1, crecimiento mixto aguas abajo y línea central y b) Caso 2, crecimiento aguas abajo

\section{Sismo de análisis}

La severidad de un sismo se encuentra reflejada en la intensidad y duración del mismo, ambos en general se incrementan con la magnitud del sismo. Adicionalmente, el contenido de frecuencias del sismo juega un rol importante en el comportamiento de presas. El movimiento puede ser amplificado fuertemente si la frecuencia predominante del sismo coincide con la frecuencia fundamental de la presa y su fundación. Para este caso de estudio se ha considerado un sismo del tipo subductivo que presenta una aceleración máxima de $0.57 \mathrm{~g}$ y una duración de $98 \mathrm{~s}$. En la Figura 3 se presentan las principales características del sismo de análisis.

\section{Caracterización geotécnica de los materiales}

En la Tabla 1 se resumen los parámetros geotécnicos considerados para los materiales involucrados en la modelación. Se ha considerado de forma simplificada que el suelo de fundación corresponde a roca. Para el muro de

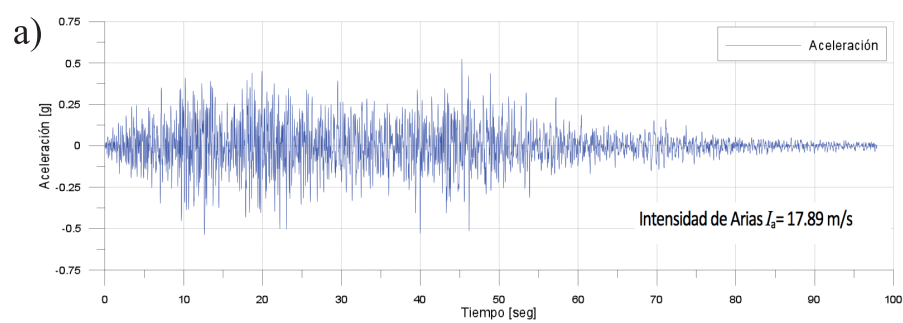

b)

c)
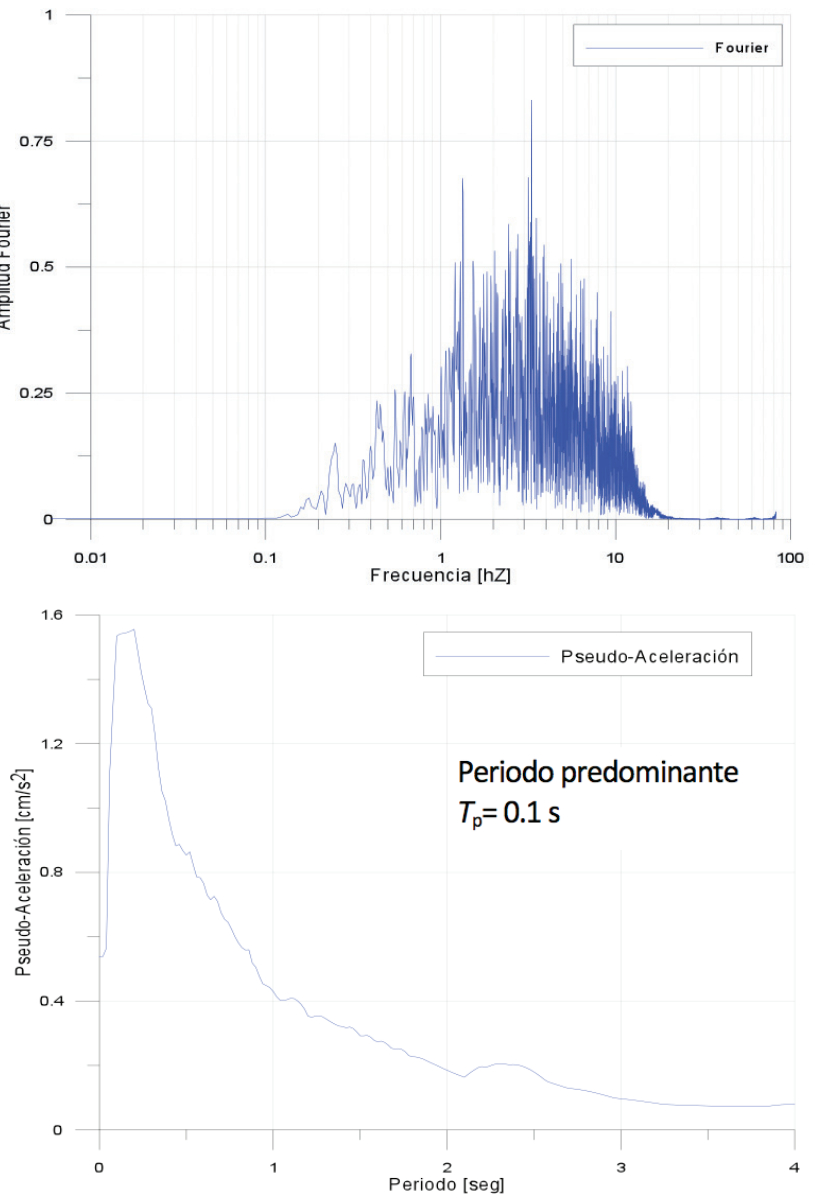

Figura 3: Sismo considerado en el análisis. a) Registro de aceleraciones, b) espectro de Fourier y c) respuesta de pseudo aceleraciones

Tabla 1: Parámetros geotécnicos adoptados

\begin{tabular}{|l|c|c|c|c|c|}
\hline \multicolumn{1}{|c|}{ Parámetro } & Unidad & Suelo de fundación & Muro de partida & Arenas de relaves & Lamas \\
\hline Peso unitario & $\mathrm{kN} / \mathrm{m}^{3}$ & 22 & 20 & 18 & 18 \\
\hline Ángulo de fricción interna $Y$ & ${ }^{3}$ & 35 & 38 & 35 & 30 \\
\hline Cohesión $c$ & $\mathrm{kPa}$ & 0 & 0 & 0 & 0 \\
\hline Módulo de deformación estático $E_{\mathrm{s}}{ }^{(1)}$ & $\mathrm{kPa}$ & $95000(\mathrm{Z})^{0.35}$ & $900\left(\sigma_{\mathrm{c}}{ }^{0.5}\right.$ & $720\left(\sigma_{\mathrm{c}}{ }^{0.5}\right.$ & $60\left(\sigma_{\mathrm{c}}{ }^{0.5}\right.$ \\
\hline Módulo de deformación dinámico $E_{\mathrm{d}}{ }^{(1)}$ & $\mathrm{kPa}$ & $3 E_{\mathrm{s}}$ & $3 E_{\mathrm{s}}$ & $3 E_{\mathrm{s}}$ & $3 E_{\mathrm{s}}$ \\
\hline Resistencia no drenada $S_{\mathrm{u}}^{(1)}$ & $\mathrm{kPa}$ & - & - & $0.27 \sigma_{\mathrm{v}}{ }^{\prime}$ & $0.05 \sigma_{\mathrm{v}}{ }^{\prime}$ \\
\hline \multirow{2}{*}{ Razón de Poisson $v$} & - & \multirow{2}{*}{0.3} & 0.25 & 0.3 (condición drenada) \\
\cline { 4 - 6 } & & & & 0.49 (condición no drenada) \\
\hline
\end{tabular}

(1) Presión de confinamiento efectiva, expresada en $\mathrm{kPa}$ y $\mathrm{Z}$ en $\mathrm{m}$ medido desde nivel de superficie 
partida se consideraron parámetros típicos de materiales de empréstito. La caracterización de las lamas se efectuó considerando los datos reportados en la literatura (RojasGonzález et al., 1985).

Campaña et al. (2013) estudiaron el comportamiento de las arenas de relave de yacimientos de cobre en Chile y Perú, a distintas presiones de confinamiento y con un variado contenido de finos. A partir de ensayos triaxiales se concluyó que el ángulo de fricción interna de las arenas de relave varía típicamente entre $32^{\circ}$ a $36^{\circ}$, con una cohesión nula. En este caso, se ha considerado un valor del ángulo de fricción de $35^{\circ}$. Para la definición de la resistencia no drenada y módulo de deformación de las arenas, se consideraron los resultados presentados por Campaña et al. (2013) y Verdugo (2011). Para estos materiales se utilizó un modelo elasto-plástico con criterio de falla o plasticidad del tipo Mohr-Coulomb.

\section{Análisis dinámico}

Para evaluar la estabilidad estática y sísmica del muro principal, se ha utilizado la herramienta computacional FLAC 3D (2016) versión 5.0. FLAC 3D corresponde a un programa computacional que permite realizar un análisis tensión - deformación bidimensional y tridimensional en masas de suelo y estructuras sometidas a distintos estados de carga estáticos y sísmicos. Esta herramienta se basa en la discretización de una masa de suelo a través de una malla de diferencias finitas, para posteriormente resolver las ecuaciones de equilibrio estático y dinámico.

\section{Nivel freático}

Para los análisis desarrollados se ha adoptado como criterio que la laguna de clarificación se encuentra lejana al muro, por lo que consecuentemente, el nivel freático se deprime rápidamente en las cercanías del muro de arenas. Según Valenzuela (2015), para que se produzca este efecto es fundamental que la permeabilidad de las arenas supere en 2 a 3 órdenes de magnitud a las permeabilidades de los relaves y/o lamas depositados y en contacto con la presa. Con este contraste se garantiza que el flujo de agua proveniente de los relaves depositados y, principalmente, del agua de transporte y depositación de las arenas, sea lo más próximo a un flujo gravitacional en un medio no saturado, alcanzando rápidamente los drenes basales de la presa. Para efectos del presente trabajo, se ha considerado que el nivel freático se deprime en el contacto de los relaves con el muro de arena, y tiene una altura de $10 \mathrm{~m}$ en la base del muro. Dichos valores son concordantes con lo reportado en la literatura para presas de relaves (Ortiz et al., 2012, Castro, 2003, Illanes et al., 2015). Bajo el nivel freático se considerará que las arenas y relaves (lamas) han licuado desarrollando la resistencia no drenada residual, una vez que se gatilla el sismo.

\section{Características geométricas de la sección y condiciones de borde}

Se definen las dimensiones del modelo con objeto de evitar la influencia de los bordes en los resultados del análisis en la zona de la presa. Para lograr esta condición, se considera una extensión horizontal de $3600 \mathrm{~m}$ y una profundidad de terreno de fundación de $650 \mathrm{~m}$. La presa se emplaza de manera que los límites de su fundación se ubiquen aproximadamente equidistantes de los bordes.

La malla de diferencias finitas se discretiza de modo de permita una adecuada propagación de las ondas para las frecuencias sísmicas relevantes del análisis dinámico. Para ello, la altura de los elementos de la malla se limitó de acuerdo a las sugerencias de Kuhlemeyer y Lysmer (1973). La distancia mínima vertical entre nodos de una malla $L_{\mathrm{m}}$, se obtiene de acuerdo a las siguientes expresiones:

$$
\begin{aligned}
& L_{\mathrm{m}} \leq \frac{\lambda}{8} \\
& L_{\mathrm{m}} \leq \frac{V_{s}}{8 f}
\end{aligned}
$$

donde $\lambda$ es la longitud de onda, $V_{\mathrm{s}}$ es la velocidad de propagación de onda de corte, y $f$ es la frecuencia de interés del registro de aceleraciones. Se ha considerado el rango de frecuencias relevantes para el estudio entre 0.4 y $8 \mathrm{~Hz}$, con una frecuencia dominante en torno a $2 \mathrm{~Hz}$. En consecuencia, la altura máxima de los elementos que componen la malla son del orden de $4 \mathrm{~m}$ para la presa, y de $10 \mathrm{~m}$ para el suelo de fundación.

Para determinar los estados tensionales estáticos en las etapas constructivas, se han considerado condiciones de borde estándar. Esto corresponde a una base del modelo fija y bordes laterales que permiten el desplazamiento 
vertical. Para el análisis dinámico se han considerado elementos absorbentes en los bordes (free field condition) de modo de evitar ondas reflejadas que puedan modificar la respuesta en superficie.

\section{Rigidez y amortiguamiento}

El modelo constitutivo empleado (Mohr-Coulomb) no reproduce el amortiguamiento histerético de los suelos que se genera durante las solicitaciones cíclicas. En consecuencia, en el análisis dinámico, el amortiguamiento se simula matemáticamente como una componente en las ecuaciones de equilibrio. Esta componente, conocida como amortiguamiento de Rayleigh, es función de las frecuencias de vibración.

El amortiguamiento Rayleigh modela el efecto combinado de un amortiguamiento proporcional a la masa $\alpha$ y otro a la rigidez $\beta$. Para un sistema con múltiples grados de libertad, se define la razón de amortiguamiento crítico $\xi_{i}$ en función de la frecuencia angular del sistema $\omega_{i}$ de la siguiente forma:

$$
\xi_{i}=\frac{1}{2}\left(\frac{\alpha}{\omega_{i}}+\beta \omega_{i}\right)
$$

Los valores de amortiguamiento se adoptan en base a curvas reportadas en la literatura técnica para distintos materiales (Rojas-González et al., 1985; Towhata, 2008), de manera que resulten compatibles con las deformaciones angulares resultantes del análisis dinámico. Los valores $\alpha$ y $\beta$ se ajustan de modo de obtener el nivel de amortiguamiento establecido, en el rango de frecuencias de interés. Con lo anterior, los valores de amortiguamiento utilizados corresponden a $2 \%$ al suelo de fundación, $3 \%$ al relave y $5 \%$ a las arenas y muro de partida.

\section{Resultados}

Los desplazamientos sísmicos remanentes horizontales y verticales se presentan en las Figura 4 y 5. Los desplazamientos máximos se localizan muy superficialmente en el talud aguas abajo, alcanzando valores del orden de $4.0 \mathrm{~m}$ y $1.8 \mathrm{~m}$ para el Caso $1 \mathrm{y}$ Caso 2, respectivamente. En el cuerpo de la presa los desplazamientos horizontales máximos resultan del orden de $3.5 \mathrm{~m}$ para el Caso 1 y $1.0 \mathrm{~m}$ para el Caso 2. Los asentamientos máximos presentan valores en torno a $0.8 \mathrm{~m}$ para el Caso $1 \mathrm{y}$ de $0.5 \mathrm{~m}$ para el Caso 2, respectivamente. En ambos casos, los valores máximos se concentran en la base de la presa, cerca del pie del talud aguas abajo. Los resultados sugieren que el desplazamiento del talud aguas abajo es fundamentalmente traslacional, probablemente propiciado por la reducción de resistencia en la zona saturada producto de la licuefacción de las arenas.

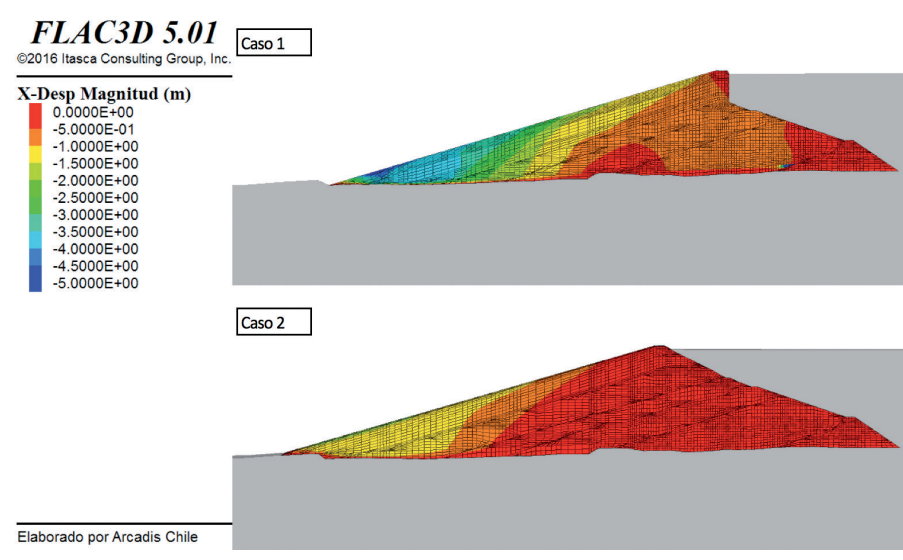

Figura 4: Análisis dinámico, desplazamientos remanentes horizontales

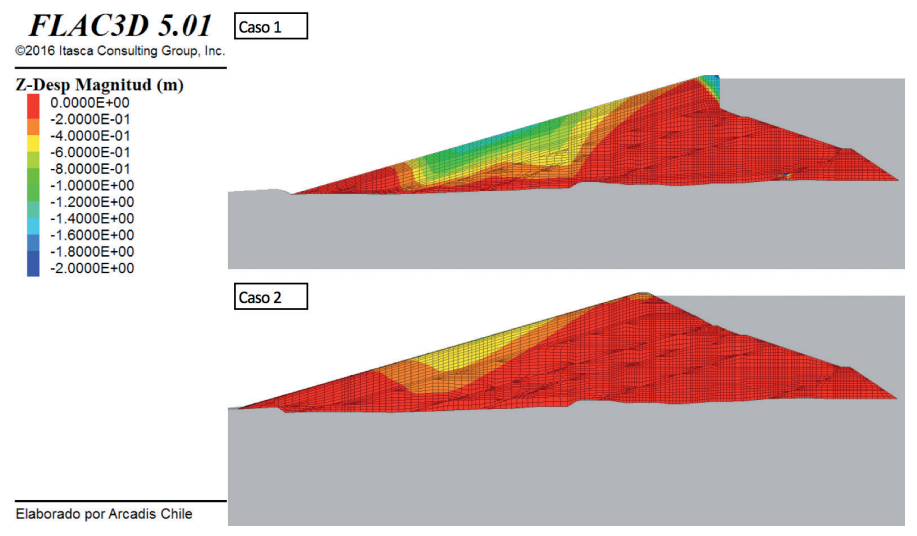

Figura 5: Análisis dinámico, desplazamientos remanentes verticales

Para el Caso 1 el coronamiento presenta desplazamientos horizontales hacia el interior de la cubeta, con valores en torno a $0.8 \mathrm{~m}$. Para el Caso 2 el coronamiento presenta desplazamientos horizontales hacia aguas abajo del orden $0.4 \mathrm{~m}$ (Figura 6). La superficie del talud aguas abajo, a mitad de altura, presenta desplazamientos horizontales remanentes de $3.0 \mathrm{~m}$ y $1.3 \mathrm{~m}$ para el Caso 1 y Caso 2 , respectivamente.

El coronamiento presenta asentamientos remanentes de $1.5 \mathrm{~m}$ y $0.25 \mathrm{~m}$ para el Caso 1 y Caso 2, respectivamente (Figura 7). La superficie del talud aguas abajo, a mitad de 


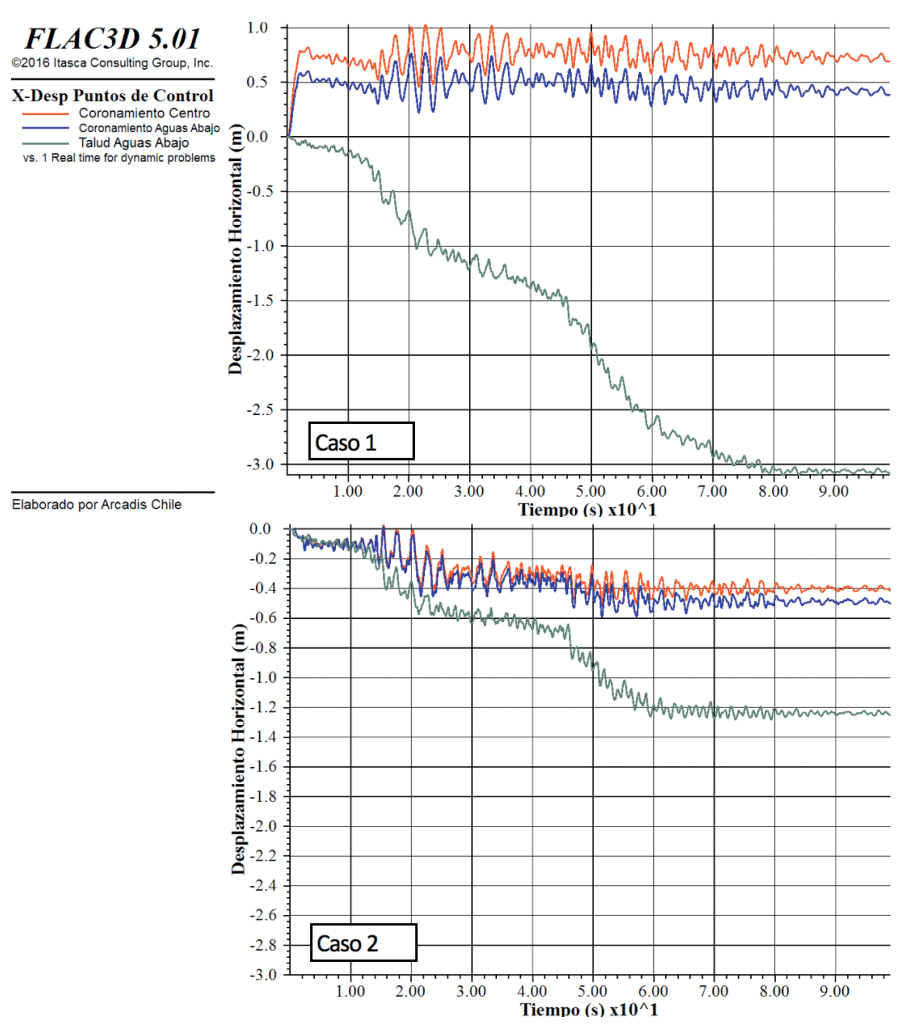

Figura 6: Desplazamientos horizontales en puntos de control

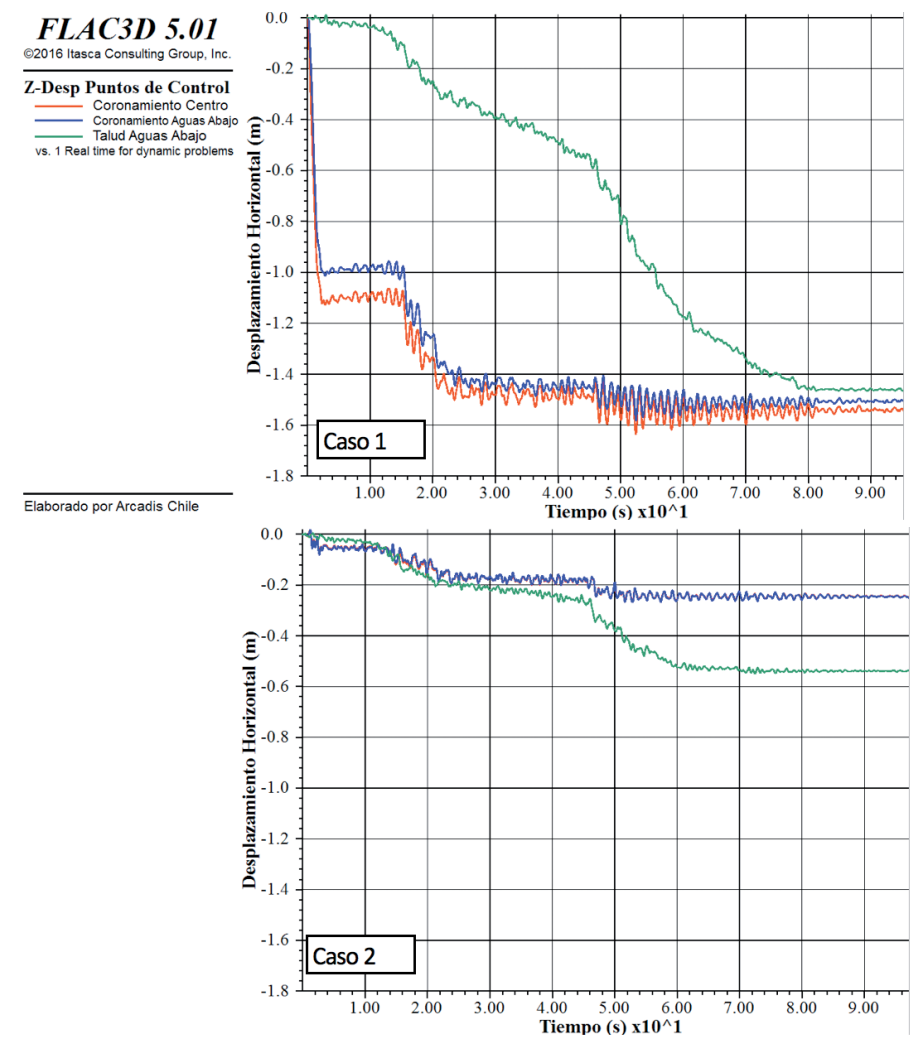

Figura 7: Desplazamientos verticales en puntos de control altura, presenta asentamientos remanentes de $1.4 \mathrm{~m}$ para el Caso 1. Estos valores resultan menores para el Caso 2, del orden de $0.55 \mathrm{~m}$.

El Caso 1 presenta un deslizamiento del talud aguas arriba, lo cual resulta esperable para el crecimiento en línea central y la magnitud del sismo empleado. Sin embargo, esta condición no compromete la estabilidad global de la presa.

En la Figura 8 se presentan los historiales de aceleraciones horizontales determinados en los puntos de control de coronamiento, suelo de fundación en campo libre, y base de la presa. En ambos casos se obtiene un correcto ajuste de PGA en campo libre. En el coronamiento se alcanza una aceleración máxima de $0.58 \mathrm{~g}$ para el Caso 1 , que determina un factor de amplificación de 1.0. Para el Caso 2 se tiene una aceleración máxima de $0.66 \mathrm{~g}$, que resulta corresponde a un factor de amplificación sísmica de 1.15 con respecto a campo libre.

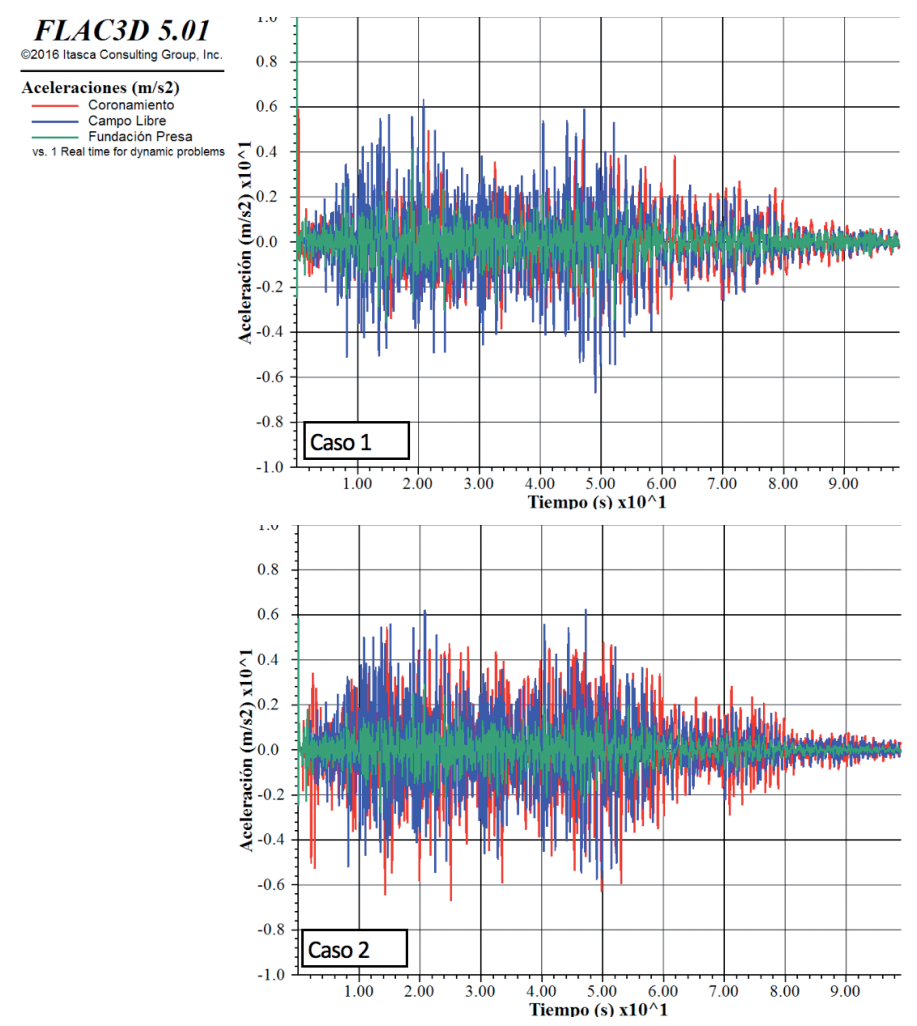

Figura 8: Aceleraciones horizontales en puntos de control

En la Figura 9 se presenta la función de transferencia entre el Coronamiento/Base Presa y Coronamiento/ Campo Libre. Los resultados indican que la frecuencia fundamental de la presa para el Caso 1 se sitúa en torno a 
$0.9 \mathrm{~Hz}$, mientras que para el Caso 2 se encuentra en torno a $1.8 \mathrm{~Hz}$. Las variaciones en la función de transferencia se asocian principalmente al efecto conjunto de la modificación geométrica y distinto nivel de deformaciones que se produce en el coronamiento.

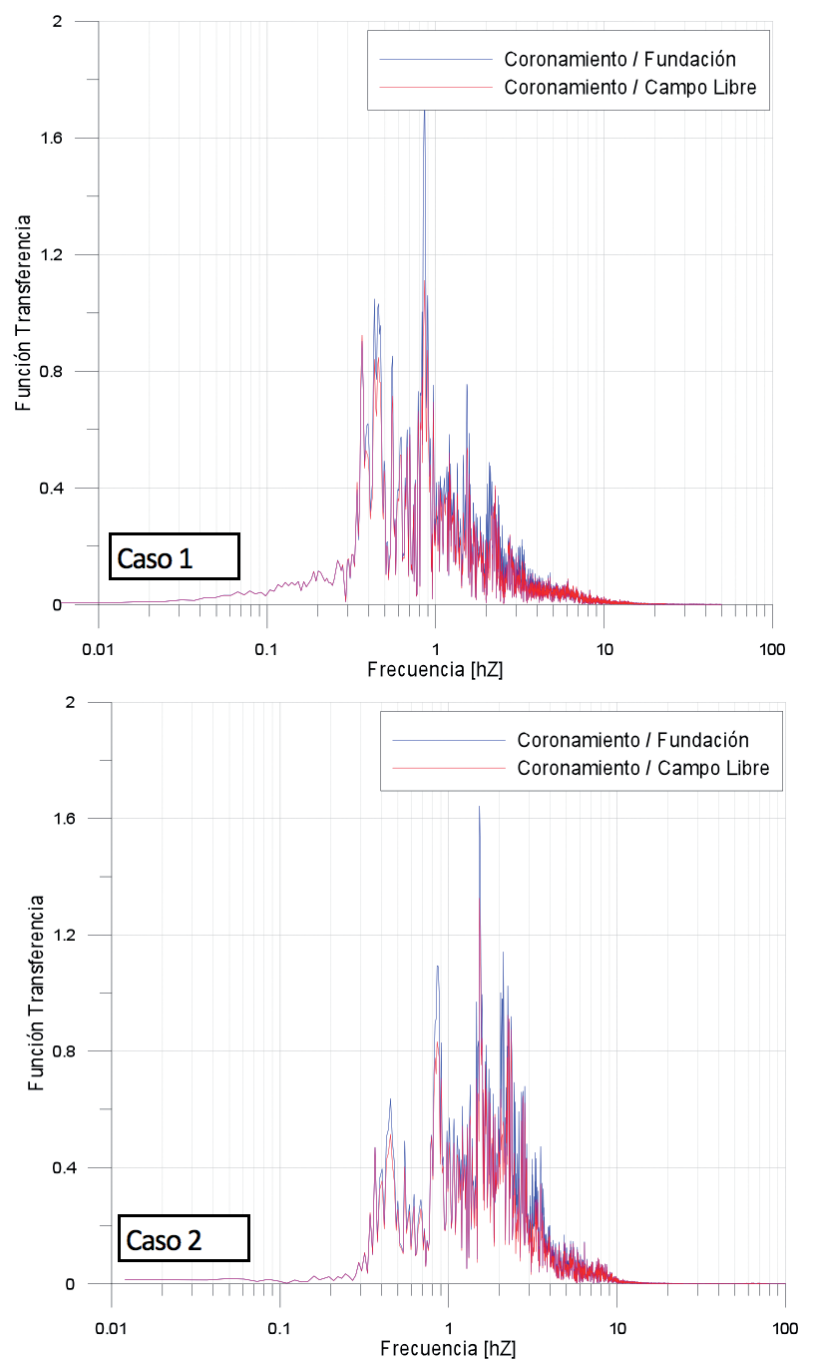

Figura 9: Función de transferencia de puntos de control

\section{Comentarios finales}

En este artículo se analizan dos geometrías de presas de arenas de relave, cuyas configuraciones corresponden a un crecimiento aguas abajo y otro mixto (aguas abajo y línea central). Se evaluó la respuesta sísmica para ambos casos, obteniéndose las siguientes conclusiones:

En ambos casos, los desplazamientos máximos se concentran en la base de la presa, cerca del pie del talud aguas abajo. Los resultados sugieren que el desplazamiento del talud aguas abajo es fundamentalmente traslacional, propiciado por la reducción de resistencia basal en la zona saturada que presenta licuefacción.

Los desplazamientos para la geometría mixta (Caso 1: aguas abajo y luego línea central) resultaron mayores que para la geometría aguas abajo (Caso 2). Esto se explica en gran medida debido a que el cuerpo resistente de la presa es menor en el Caso 1.

Respecto a la respuesta de aceleraciones en el coronamiento, en el Caso 1 no se presenta una amplificación de aceleraciones respecto al campo libre, presumiblemente por el nivel de deformaciones que presenta el coronamiento. En cambio, el Caso 2 presenta una amplificación de aceleraciones del orden de 1.15.

Para el Caso 1 se evidencia un deslizamiento del coronamiento de la presa hacia aguas arriba. Esta condición no se presenta para el Caso 2. Esta situación debe ser considerada en el diseño de este tipo de presas, con el fin de asegurar un ancho de coronamiento mínimo post sismo.

La función de transferencia se ve modificada al cambiar el método de crecimiento comparando las alternativas estudiadas, aumentando el periodo fundamental de la presa de $0.9 \mathrm{~Hz}$ para el caso mixto a $1.8 \mathrm{~Hz}$ para el caso aguas abajo.

Los resultados indican que la presa del tipo aguas abajo presenta un mejor comportamiento ante eventos sísmicos, lo que resulta consecuente con lo señalado en la literatura técnica (Vick, 1983).

\section{Referencias}

Campaña, J., Bard, E. and Verdugo, R. (2013). Shear strength and deformation modulus of tailing sands under high pressures. $18^{\text {th }}$ Conference on Soil Mechanics and Geotechnical Engineering, Paris

Castro, G. (2003). Evaluation of seismic stability of tailings dams. XII Pan-American Conference on Soil Mechanics and Geotechnical Engineering, Cambridge, USA, 16-23

FLAC 3D (2016). Fast Lagrangian Analysis of Continua. Version 5.0. Itasca Consulting Group, Inc. Minneapolis: Itasca

Illanes, J. Urquidi, J. Figueroa, A. Campaña, J. and Morales, F. (2015). Geotechnical instrumentation in tailings sand dams. XV Pan-American Conference on Soil Mechanics and Geotechnical Engineering, Buenos Aires 
Kuhlemeyer, R.L. and Lysmer, J. (1973). Finite element method accuracy for wave propagation problems. Journal of Soil Mechanics and Foundations Division 99(SM5), 421-427

Obermayer, J. and Alexieva, T. (2011). Design, construction and operation of a large centerline tailing storage facility with high rate of rise. Tailings and Mine Waste, Vancouver

Ortiz, J., Urquidi, J., Pollak, D. and Barrera, S. (2012). Downstream to centerline construction: the challenge of change. Tailings and Mine Waste '12, Colorado

Rojas-González, L., Ben-Khalal, H. and Lewis, K. (1985). Dynamic properties and behavior of copper tailings. XI International Conference on Soil Mechanics and Foundation Engineering, San Francisco, California, USA, 3: 1289-1292

Scott, M.D., Lo, R.C. and Thavaraj, T. (2007). Use of instrumentation to safeguard stability of a tailings Dam. $7^{\text {th }}$ International Symposium on Field Measurements in Geomechanics, ASCE, 1-13
Towhata, I. (2008). Geotechnical earthquake engineering. Springer

Valenzuela, L. (2015). Tailings dams and hydraulic fills. Casagrande lecture. XV Pan-American Conference on Soil Mechanics and Geotechnical Engineering, Buenos Aires Verdugo, R. (2011). Seismic stability analysis of large tailings dams. $5^{\text {th }}$ International Conference on Earthquake Geotechnical Engineering, Santiago, 359-383

Vick, S.G. (1983). Planning, design and analysis of tailings dams. John Wiley \& Sons 